\title{
Optimizing Post Activation Potentiation for Explosive Activities in Competitive Sports
}

\author{
by

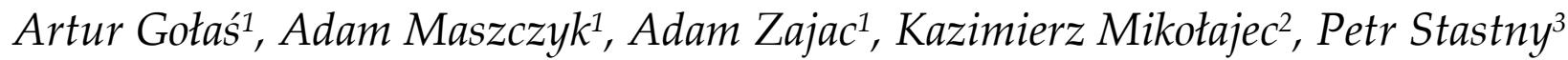

Post activation potentiation (PAP) has shown improved performance during movements requiring large muscular power output following contractions under near maximal load conditions. PAP can be described as an acute enhancement of performance or an enhancement of factors determining an explosive sports activity following a preload stimulus. In practice, PAP has been achieved by complex training, which involves a combination of a heavy loaded exercise followed by a biomechanically similar explosive activity, best if specific for a particular sport discipline. The main objective of this study was to investigate the effects of PAP on performance in explosive motor activities specific for basketball, luge and athletics throws. The novel approach to the experiments included individualized recovery time (IRT) between the conditioning exercise and the explosive activity. Additionally, the research groups were homogenous and included only competitive athletes of similar age and training experience. Thirty one well trained athletes from 3 different sport disciplines participated in the study. All athletes performed a heavy loaded conditioning activity (80130\%1RM) followed by a biomechanically similar explosive exercise, during which power $(W)$ or the rate of power development $(\mathrm{W} / \mathrm{s} / \mathrm{kg}$ ) was evaluated. The results of our experiment confirmed the effectiveness of PAP with welltrained athlets during explosive motor activities such as jumping, throwing and pushing. Additionally, our research showed that eccentric supramaximal intensities (130\% 1RM) can be effective in eliciting PAP in strength trained athletes. Our experiments also showed that the IRT should be individualized because athletes differ in the strength level, training experience and muscle fiber structure. In the three experiments conducted with basketball players, track and field athletes and luge athletes, the optimal IRT equaled 6 min. This justifies the need to individualize the volume and intensity of the $C A$, and especially the IRT, between the $C A$ and the explosive activity.

Key words: power, athletes, complex training, conditioning exercise.

\section{Introduction}

There are numerous sport disciplines and events in which performance is highly influenced by the rate of power development (RPD). These include track \& field sprints, throws, jumps and hurdling, as well as most team sport games and combat sports. Besides anthropometric characteristics and proper technique, explosive strength is a prerequisite of high performance in these sports. A phenomena known as the post activation potentiation (PAP) has shown improved performance during movements requiring large muscular power output following contractions under near maximal load conditions (Gourgoulis et al., 2003; Mcbride et al., 2005; Young et al., 1998). PAP can be described as an acute enhancement of performance or an enhancement of factors determining an explosive sports activity following a preload stimulus (Whelan et al., 2014). In practice, PAP has been achieved by complex training, which involves a combination of a heavy loaded exercise followed by a biomechanically similar explosive activity,

\footnotetext{
1 - Department of Sports Training; The Jerzy Kukuczka Academy of Physical Education in Katowice; Poland.

2 - Department of Team Sport Games; The Jerzy Kukuczka Academy of Physical Education in Katowice; Poland.

3 - Charles University in Prague, Faculty of Physical Education and Sport.
} 
best if specific for a particular sport discipline (Weber et al., 2008). The presence of PAP has been documented in many studies in both mammals and humans, which allowed to pinpoint the mechanisms underlying this phenomenon (Guillich and Schmidtbleicher, 1996; Stuart et al., 1988; Tillin and Bishop, 2009; Vandervoort et al., 1983). Scientists have indicated to practitioners that PAP if effectively utilized, could be implemented into power training routines to enhance the training stimulus of explosive strength exercises (Docherty and Hodgson, 2007; French et al., 2003; Magiera et al., 2013; Stastny et al., 2016; Yetter and Moir, 2008). Others have proposed to include PAP prior to competition in warm-up routines to enhance performance of explosive sport activities such as jumping, throwing and sprinting (Docherty and Hodgson, 2007; Gullich and Schmidtbleicher, 1996). Despite numerous studies, inconsistencies within the literature regarding the possible benefits of PAP to explosive sport performance remain inconclusive. The inconsistencies of previous research stem from the complex interaction of factors that influence acute performance following conditioning contractions (CC) (Tillin and Bishop, 2009).

Different mechanisms have been proposed to cause PAP, yet two seem to be well justified by research. One includes the phosphorylation of myosin light chains resulting from the initial muscle activity, which would render the actin and myosin molecules more sensitive to $\mathrm{Ca} 2+$ availability, what is related to increased rates of force development and maximal isometric force (Baudry and Duchateu, 2007; Czuba et al., 2013; Hodgson et al., 2005; Sale, 2002, 2004). The other mechanism of PAP includes neural factors, such as the excitability of $\alpha-$ motoneurons as being responsible for increased contractile performance after previous muscular activity. It has been proposed that a high load conditioning exercise increases higher order motoneuron recruitment, what theoretically may increase fast-twitch fiber contribution, and thus enhance performance in subsequent explosive activities (Trimble and Harp, 1998; Yetter and Moir, 2008). A third mechanism underlying PAP has also been proposed, yet it has not been studied as extensively as the two others mentioned above. Some evidence suggests that changes in the pennation angle may contribute to PAP. The pennation angle of a muscle reflects the orientation of muscle fibers in relation to connective tissue and tendons. The pennation angle will therefore affect force transition to the tendon and bones. Some research has confirmed that smaller pennation angles have a mechanical advantage with respect to force transmission to the tendon (Folland and Willimas, 2007; Fukunaga et al., 1997).

The effects of PAP have been ubiquitously reported in studies using different modes of the conditioning contraction (CC) or conditioning activity (CA) (Hodgson et al., 2005; Tillin and Bishop, 2009). However, a consistent and significant finding of these studies is the high inter-individual variability in the voluntary PAP response. Increases (Baker, 2003; French et al., 2003; Mangus et al., 2006; Wilson et al., 2013), no change (Gossen and Sale, 2000; Gourgoulis et al., 2003; Jensen and Eben, 2003), as well as decreases (Chiu et al., 2003; Duthie et al., 2002) in muscular performance after the CA have been reported, what is indicative of a responder versus nonresponder phenomenon. Previous research has indicated that there are numerous factors that modulate PAP and its effect on performance in subsequent explosive activities. These may include the type of CA, its intensity and volume, and most of all the recovery period between the $\mathrm{CA}$ and the explosive movement. The similarity between the CA and the subsequent movement also seems important, as some authors have indicated better effects of PAP when a heavy loaded exercise $(80-90 \% 1 \mathrm{RM})$ is followed by a biomechanically similar explosive activity (Whelan et al., 2014), and that the CA's range of motion plays a role in subsequent performance (Esformes and Bampouras, 2013; Seitz et al., 2015). Subject characteristics may also be an important factor when considering PAP effectiveness. Several studies have shown that well trained, stronger athletes show greater PAP susceptibility (Seitz et al., 2014). This indicates that subjects with a high proportion of type II muscle fibers show a better PAP response (Chiu et al., 2003; Hamada et al., 2000, 2003). Subject characteristics have varied tremendously throughout particular research projects, including youth and adult subjects, recreationally active subjects to elite athletes, female and male subjects as well as 
representatives of different sport disciplines. Because the above mentioned variables have not been standardized across past research, the effects of CA on performance of subsequent explosive activities are inconclusive and require further research.

The main objective of this study was to investigate the effects of PAP on performance in explosive motor activities specific for 3 different sport disciplines, including basketball, luge and athletics throws. The novel approach to the experiments included individualized recovery time between the conditioning exercise and the explosive activity. Additionally, the research groups were homogenous and included only competitive athletes of similar age and training experience.

\section{Material and Methods}

\section{Experimental approach to the problem}

Effects of PAP on performance in explosive motor activities in the literature are widely described, but still there are many unknowns. Most of the data applies to recreational athletes and such knowledge is not always useful for competitive sports training. In addition, there is no information on supramaximal loads (110-150\% 1RM) used as the conditioning activity, and the data on the optimal recovery time between the conditioning activity and explosive movement is highly inconclusive.

\section{Subjects}

Thirty-one $(\mathrm{n}=31)$ well trained athletes participated in this study. The research involved athletes from 3 different sport disciplines:

1. Basketball players $(n=13$; body height: $195 \pm 8.3 \mathrm{~cm}$; body mass: $87.10 \pm 15.62 \mathrm{~kg}$; age: 25.3 \pm 3.6 years; training experience: $11 \pm 2.3$ years).

2. Athletics throws (shot putters, javelin throwers, discuss throwers) $(\mathrm{n}=10$; body height: $188 \pm 6.9 \mathrm{~cm}$, body mass: $105.10 \pm 11.62 \mathrm{~kg}$; age: $28.7 \pm 4.6$ years; training experience: $13 \pm 4$ years).

3. Luge athletes - National Team $(n=8$; body height $183 \pm 4.7 \mathrm{~cm}$; body mass $82.5 \pm 5.3 \mathrm{~kg}$, age $26 \pm 2$ years; training experience: $7.9 \pm 2$ years).

The participants did not perform any additional resistance training 72 hours prior to testing. All the subjects were informed verbally and in writing about the procedures, as well as the possible risks and benefits of the tests and provided written consent before they were included in the study. The study received the approval of the Bioethics Committee at the Academy of Physical Education in Katowice, Poland.

\section{Experimental Protocol}

Protocol for Basketball players

The measurements were performed in the Strength and Power Laboratory at the Academy of Physical Education in Katowice. Testing was carried out during two sessions. The first, preliminary session was used to determine the $1 R M$ value and to establish the Individual intracomplex Rest Interval (IRT). The 1-RM load achieved during the Squat exercise was determined using the protocol outlined by Baechle et al. (2008). The subjects were expected to reach their 1RM effort within 3-5 maximal attempts (Thomas et al., 2007). The IRT Squat Test was carried out on the Keiser Squat machine. The test was performed with an explosive movement with the load set at 60\% 1RM. The players performed 3 sets of 8 reps with a load of $75 \% 1 R M$ and $90 \mathrm{~s}$ rest periods between each set. After a 2, 4,6 and $8 \mathrm{~min}$ rest interval the subjects performed the explosive movement with the load of $60 \%$ $1 \mathrm{RM}$.

The second, main experimental session began after 7 days of recovery and was timed as follows: Pre-conditioning (twice Counter Movement Jump), conditioning protocol (4 sets of 4 reps of the Keiser Squat at $80 \% 1 \mathrm{RM}$ with $90 \mathrm{~s}$ rest between sets), followed by IRT postconditioning (twice Counter Movement Jump). The Rate of Power Development during the CMJ was measured on a force plate (Accu Power) under functional conditions (for later analysis the better result of the two measurements was used). The rest between sets during the conditioning activity and explosive movement complied with previously determined values of IRT (Table 1).

Protocol for athletics throws

The evaluations were carried out in two sessions separated by 7 days of recovery. The first session was used to determine the 1RM value and to establish the Individual intra-complex Rest Interval (IRT). The participants followed the same warm up protocol as used by Saeterbakken et al. (2011). They started with a regular warm up procedure of the shoulders and arms followed by four warm-up sets of the bench press: 1) twenty 
repetitions at $30 \%$ of $1 \mathrm{RM}, 2)$ twelve repetitions at $50 \%$ of $1 \mathrm{RM}, 3$ ) six repetitions at $70 \%$ of $1 \mathrm{RM}$, and 4 ) one repetition at $85 \%$ of $1 \mathrm{RM}$ were performed. The percentage of the 1RM was estimated according to the protocol used by Tillar and Ettema (2013). The IRT Test was carried out on the Keiser Power Rack machine with the Bench Press Throw. The test was performed with an explosive movement with a 50\% 1RM load, followed by 3 sets of 8 reps with a load of $75 \% 1 \mathrm{RM}$. After 2, 4, 6 and 8 minute rest intervals, the athletes repeated the explosive movement with $50 \% 1 \mathrm{RM}$ to determine the optimal recovery time. The second testing session was carried out on a Smith machine using the Bench Press Throw, with the measurements performed by a three-dimensional accelerometer (Myotest Pro, Myotest S.A., France). The conditioning activity consisted of 2 sets of 3 reps of the flat bench press with free weights with a load of 110 and $130 \% 1 \mathrm{RM}$, and a rest period of 4-5 min between sets. The rest interval between the conditioning activity and the explosive movement complied with the previously determined value of the IRT. Between the $110 \%$ $1 \mathrm{RM}$ and $130 \%$ 1RM the participants rested for 15 minutes to avoid fatigue. The athletes were asked to execute each bench press repetition at a specific tempo (approximately $2 \mathrm{~s}$ for the eccentric phase) and use full range of motion. Spotters were present at all time to verbally encourage the subjects and to ensure their safety. The participants of this experiment were very experienced with resistance training, did not report any subjective evidence of musculoskeletal disorders and demonstrated a 1RM bench press $(120-150 \%$ BM) significantly in excess of their body mass (Farup and Sorensen, 2010).

Protocol for Luge

The measurements were performed in the Laboratory of Muscular Force and Power at the Academy of Physical Education in Katowice and on the Königssee luge track in Germany. There were three sessions of the experiment. The first session was comprised of two runs on the luge track in order to determine the best start time (push and paddle). The second session was used to determine the $1 \mathrm{RM}$ value in the Latissimus pull down exercise and flat bench press on the Keiser Power Rack and to establish the Individual intracomplex Rest Interval (IRT). The participants followed the same warm up protocol as used by
Saeterbakken et al. (2011).

The percentage of the 1RM was estimated according to the protocol used by Tillar and Ettema (2013). The IRT Test was carried out on the Keiser Power Rack with the Bench Press Throw. The test was performed with an explosive movement with a $50 \% 1 \mathrm{RM}$ load and then the subjects performed 3 sets of 8 reps with a load of $75 \%$ 1RM. After 2, 4, 6 and 8 minute rest intervals, the athletes repeated the explosive movement with $50 \% 1 \mathrm{RM}$ to determine the optimal recovery time. During the third session we attempted to determine the impact of the Latissimus pull down exercise (CA) performed on the Keiser Power Rack on the luge start. The experimental conditions were timed as follows: Preconditioning (2 Latissimus pull down repetitions on Keiser Power Rack at 50\% 1RM), conditioning protocol (3 sets of 4 reps dumbbell row at $80 \%$ $1 \mathrm{RM}$ with a $90 \mathrm{~s}$ rest interval), followed by IRT post-conditioning (2 Latissimus pull down repetitions on Keiser Power Rack at 50\% 1RM).

\section{Statistical Analysis}

Normality of distribution was verified using the Shapiro-Wilk test. Verification of the differences between the Rate Power Development $(\mathrm{W} / \mathrm{s} / \mathrm{kg})$ during the CMJ, Power $(\mathrm{W})$ generated during the explosive bench throw on a Smith Machine and Power (W) generated during the pull down on the Keiser Power Rack, after activation was carried out using the ANOVA analysis of variance. Between power generated during the Keiser pull down and the luge start, correlation coefficients were determined with Pearson product-moment correlation coefficients. Statistical significance was set at $p<0.05$. All statistical analyses were performed using Statistica and Microsoft Office Excel software.

\section{Results}

The values of power generated after activation including the rest interval, in particular groups of athletes are presented in Tables 1 to 3 .

The ANOVA revealed statistically significant effects of rest intervals on power with $p$ $=0.014$ for $2 \mathrm{~min}, p=0.036$ for $4 \mathrm{~min}, p=0.001^{*}$ for $6 \mathrm{~min}$ and $p=0.036$ for $8 \mathrm{~min}$. For all 3 sport disciplines the 6 min rest interval was the most optimal (Table 4).

The values of the rate of Power Development during the CMJ (basketball) and 
Power $(\mathrm{W})$ generated during the explosive bench throw on a Smith Machine (athletics throws) before and after activation with the eccentric flat bench press performed with loads of $110 \%$ and $130 \% 1 \mathrm{RM}$ are presented in Tables 5 and 6 .

The results of ANOVA revealed a significant effect of activation on the Rate of Power Development $(\mathrm{W} / \mathrm{s} / \mathrm{kg})$ during the CMJ before and after activation with $\mathrm{F}=14.84$ and $p=$ 0.037 .

Similarly, ANOVA revealed statistically significant differences in Power generated during the bench throw on a Smith Machine before and after activation with the eccentric flat bench press performed with loads of 130\% 1RM ( $\mathrm{F}=16.64 ; p=$ 0.018). The changes in generated power during the bench throw after the eccentric flat bench press with a load of $110 \% 1 \mathrm{RM}$ were statistically nonsignificant $(\mathrm{F}=4.10 ; p=0.057)$.
The analysis of Pearson product-moment correlation coefficients revealed very high, statistically significant negative correlations between power generated during the Keiser Latissimus pull down and the luge start with $r=-$ 0.87 (Table 7).

The values of Power (W) generated during the Latissimus pull down on Keiser Power Rack at 50\% 1RM before and after activation with the dumbbell row at $80 \%$ 1RM activation are presented in Table 8.

The results of ANOVA revealed statistically significant differences in Power generated during the pull down on Keiser Power Rack at 50\% 1RM before and after activation with the dumbbell row at $80 \% 1 \mathrm{RM}(\mathrm{F}=15.40 ; p=$ 0.035).

Table 1

Power generated during the Keiser Squat $(W)$ before and after activation

\begin{tabular}{|c|c|c|c|c|c|}
\hline \multicolumn{6}{|c|}{ Basketball players $(n=13)$} \\
\hline \multicolumn{2}{|c|}{$\begin{array}{c}\text { Power generated before } \\
\text { activation }(\mathrm{W})\end{array}$} & \multicolumn{4}{|c|}{ Power generated after activation (W) } \\
\hline & & $2 \mathrm{~min}$ & $4 \mathrm{~min}$ & $6 \mathrm{~min}$ & $8 \mathrm{~min}$ \\
\hline 1 & 1431 & 1430 & 1452 & $1491^{*}$ & 1465 \\
\hline 2 & 1583 & 1601 & $1611^{*}$ & 1595 & 1593 \\
\hline 3 & 1724 & 1692 & 1710 & $1755^{*}$ & 1731 \\
\hline 4 & 1948 & 1931 & 1952 & $1970^{*}$ & 1929 \\
\hline 5 & 1750 & $1765^{*}$ & 1761 & 1760 & 1755 \\
\hline 6 & 1833 & 1790 & 1821 & 1833 & $1864^{*}$ \\
\hline 7 & 1680 & 1675 & 1702 & $1730^{*}$ & 1721 \\
\hline 8 & 1626 & 1619 & 1631 & $1659^{*}$ & 1632 \\
\hline 9 & 1891 & 1854 & $1910^{*}$ & 1893 & 1888 \\
\hline 10 & 1620 & 1644 & $1678^{*}$ & 1654 & 1658 \\
\hline 11 & 1730 & 1717 & 1711 & $1741^{*}$ & 1719 \\
\hline 12 & 1745 & 1739 & 1743 & $1787^{*}$ & 1769 \\
\hline 13 & 1936 & 1911 & $1945^{*}$ & 1931 & 1925 \\
\hline
\end{tabular}

${ }^{*} p<0.05$ versus power generated before activation 
Table 2

Power generated during the flat bench press on the Keiser Power Rack (W) before and after activation in field athletes

\begin{tabular}{|c|c|c|c|c|c|}
\hline \multicolumn{6}{|c|}{ Athletics throws $(n=10)$} \\
\hline \multicolumn{2}{|c|}{$\begin{array}{c}\text { Power generated before } \\
\text { activation }(\mathrm{W})\end{array}$} & \multicolumn{4}{|c|}{ Power generated after activation $(\mathrm{W})$} \\
\hline & & $2 \mathrm{~min}$ & $4 \mathrm{~min}$ & $6 \mathrm{~min}$ & $8 \mathrm{~min}$ \\
\hline 1 & 713 & 670 & 700 & $751^{*}$ & 686 \\
\hline 2 & 815 & 790 & 809 & 810 & $865^{*}$ \\
\hline 3 & 630 & 635 & $645^{*}$ & 632 & 631 \\
\hline 4 & 890 & 879 & 887 & $920^{*}$ & 890 \\
\hline 5 & 925 & 905 & 928 & $940^{*}$ & 890 \\
\hline 6 & 810 & 790 & 805 & 801 & $825^{*}$ \\
\hline 7 & 720 & 735 & $756^{*}$ & 751 & 711 \\
\hline 8 & 709 & 715 & 720 & $738^{*}$ & 719 \\
\hline 9 & 855 & 838 & 841 & $860^{*}$ & 845 \\
\hline 10 & 791 & 740 & $810^{*}$ & 774 & 750 \\
\hline
\end{tabular}

Table 3

Power generated during the flat bench press on the Keiser Power Rack (W) before and after activation in Luge athletes

\begin{tabular}{|c|c|c|c|c|c|}
\hline \multicolumn{6}{|c|}{ Luge $(n=8)$} \\
\hline \multicolumn{2}{|c|}{$\begin{array}{c}\text { Power generated before } \\
\text { activation }(\mathrm{W})\end{array}$} & \multicolumn{4}{|c|}{ Power genrated after activation (W) } \\
\hline & & $2 \mathrm{~min}$ & $4 \mathrm{~min}$ & $6 \mathrm{~min}$ & $8 \mathrm{~min}$ \\
\hline 1 & 578 & 518 & 614 & $634^{*}$ & 610 \\
\hline 2 & 886 & 822 & 912 & 1058 & 909 \\
\hline 3 & 730 & 695 & 745 & $810^{*}$ & 795 \\
\hline 4 & 543 & 555 & 567 & $584^{*}$ & 579 \\
\hline 5 & 720 & 721 & $790^{*}$ & 750 & 744 \\
\hline 6 & 620 & 611 & 617 & 618 & $643^{*}$ \\
\hline 7 & 590 & 610 & 615 & $643^{*}$ & 630 \\
\hline 8 & 655 & 643 & 655 & $673^{*}$ & 660 \\
\hline
\end{tabular}

${ }^{*} p<0.05$ versus power generated before activation

Table 4

Individual intra-complex Rest Interval (IRT)

\begin{tabular}{lcccc}
\hline Rest interval & 2 min & 4 min & 6 min & 8 min \\
\hline Basketball players $(n=13)$ & 1 & 4 & $8^{*}$ & 1 \\
Athletics throws $(n=10)$ & 0 & 3 & $5^{*}$ & 2 \\
Luge athletes $(n=8)$ & 0 & 1 & $6^{*}$ & 1 \\
\hline
\end{tabular}


Table 5

The rate of Power Development $(\mathrm{W} / \mathrm{s} / \mathrm{kg})$ during the $\mathrm{CMJ}$ before and after activation

\begin{tabular}{|c|c|c|c|c|}
\hline \multicolumn{5}{|c|}{ Basketball players $(n=13)$} \\
\hline \multicolumn{3}{|c|}{ RPD before activation } & \multicolumn{2}{|c|}{ RPD after activation } \\
\hline & $\mathrm{W} / \mathrm{s} / \mathrm{kg}$ & SD & $\mathrm{W} / \mathrm{s} / \mathrm{kg}$ & SD \\
\hline 1 & 195 & & 265 & \\
\hline 2 & 230 & & 243 & \\
\hline 3 & 274 & & 340 & \\
\hline 4 & 343 & & 395 & \\
\hline 5 & 187 & & 212 & \\
\hline 6 & 287 & & 299 & \\
\hline 7 & 265 & 53 & 311 & 50 \\
\hline 8 & 317 & & 353 & \\
\hline 9 & 289 & & 331 & \\
\hline 10 & 269 & & 268 & \\
\hline 11 & 168 & & 254 & \\
\hline 12 & 235 & & 305 & \\
\hline 13 & 195 & & 264 & \\
\hline
\end{tabular}

Table 6

Power (W) generated during the bench throw on a Smith Machine before and after activation with the eccentric flat bench press performed with loads of $110 \%$ and $130 \% 1$ RM.

\begin{tabular}{|c|c|c|c|c|c|c|}
\hline \multicolumn{7}{|c|}{ Athletics throws $(n=10)$} \\
\hline \multicolumn{3}{|c|}{$\begin{array}{l}\text { Power generated before } \\
\text { activation }\end{array}$} & \multicolumn{2}{|c|}{$\begin{array}{c}\text { Power generated after activation } \\
\text { with } 110 \% 1 \mathrm{RM}\end{array}$} & \multicolumn{2}{|c|}{$\begin{array}{l}\text { Power generated after } \\
\text { activation with } 130 \% 1 \mathrm{RM}\end{array}$} \\
\hline & $(\mathrm{W})$ & SD & (W) & SD & (W) & SD \\
\hline 1 & 1200 & \multirow{10}{*}{127} & 1191 & \multirow{10}{*}{123} & 1310 & \multirow{10}{*}{97} \\
\hline 2 & 1296 & & 1290 & & 1398 & \\
\hline 3 & 1150 & & 1172 & & 1190 & \\
\hline 4 & 934 & & 950 & & 1188 & \\
\hline 5 & 1110 & & 1080 & & 1165 & \\
\hline 6 & 1070 & & 1075 & & 1110 & \\
\hline 7 & 1320 & & 1330 & & 1366 & \\
\hline 8 & 1095 & & 1130 & & 1299 & \\
\hline 9 & 950 & & 970 & & 1210 & \\
\hline 10 & 1150 & & 1180 & & 1345 & \\
\hline
\end{tabular}


Table 7

The relationship of power generated during the Keiser pull down with the luge start

\begin{tabular}{cccc}
\hline & Luge $(n=8)$ & \\
\hline $\begin{array}{c}\text { Power generated } \\
\text { before activation }\end{array}$ & Start Time & $\begin{array}{c}\text { Pearson product- } \\
\text { moment correlation } \\
\text { coefficient }\end{array}$ \\
\hline & $(\mathrm{W})$ & $(\mathrm{s})$ & $\mathrm{r}$ \\
1 & 578 & 3,43 & \\
2 & 886 & 3,26 & \\
3 & 560 & 3,37 & -0.87 \\
4 & 643 & 3,35 & \\
5 & 720 & 3,29 & \\
6 & 620 & 3,32 & \\
7 & 640 & 3,35 & \\
8 & 755 & 3,31 & \\
\hline
\end{tabular}

Table 8

Power (W) generated during the pull down on Keiser Power Rack at $50 \% 1 R M$ before and after activation with the dumbbell row at $80 \% 1 R M$

\begin{tabular}{|c|c|c|c|c|}
\hline \multicolumn{5}{|c|}{ Luge $(n=8)$} \\
\hline \multicolumn{3}{|c|}{ Power generated before activation } & \multicolumn{2}{|c|}{ Power generated after activation } \\
\hline & N) & $\mathrm{SD}$ & $(\mathrm{W})$ & $\mathrm{SD}$ \\
\hline 1 & 578 & \multirow{8}{*}{107} & 721 & \multirow{8}{*}{128} \\
\hline 2 & 886 & & 1045 & \\
\hline 3 & 560 & & 684 & \\
\hline 4 & 643 & & 750 & \\
\hline 5 & 720 & & 950 & \\
\hline 6 & 620 & & 775 & \\
\hline 7 & 640 & & 715 & \\
\hline 8 & 755 & & 863 & \\
\hline
\end{tabular}

\section{Discussion}

The current research, conducted on competitive athletes from 3 different sport disciplines confirmed the effectiveness of complex training in eliciting post activation potentiation of the upper and lower limbs, thus improving performance in explosive, sport specific movements. The novel aspects of this research project included the individual intra complex rest intervals (IRT), which were determined in preliminary testing sessions with an explosive movement preceded by a conditioning exercise performed in 3 sets of 8 repetitions with a load of $75 \% 1 \mathrm{RM}$. The potentiation effect varied in the 3 groups of athletes, yet for $58 \%$ of the tested subjects the most significant increase in power from pre to post activation occurred after $6 \mathrm{~min}$ from the cessation of the conditioning exercise. This is in accordance with previous studies (Whelan et al., 2014; Yetter and Moir 2008), yet the 
data shows great variability with several athletes reaching their peak power after $4 \mathrm{~min}$, most after 6 and a few as late as $8 \mathrm{~min}$ after the CA. Therefore, the estimation of the IRT for competitive athletes should be optimized, most likely using the trial and error method, experimenting with the rest interval from 2 to 10 $12 \mathrm{~min}$.

It has been established that the differences in the IRT for particular subjects and tasks, and the overall effect of contractile history on subsequent performance are influenced by several factors (Robbins, 2005). These include the volume of the CA (repetitions, sets), the intensity of the CA $(50-130 \% 1 \mathrm{RM})$, the type of contraction (dynamic, isometric, isokinetic), the type of activity (general-specific), and most of all subject characteristics, including muscular strength, training status as well as fiber type distribution (Tillin and Bishop, 2009).

The morphological factor has not been studied as extensively as the other variables determining the effectiveness of PAP, yet the literature (Tillin and Bishop, 2009) and our findings suggest its dominant role in programing PAP exercise protocols. The balance between PAP and fatigue and its effect on subsequent explosive activity has been observed in numerous studies (Hodgson et al., 2005; Whelan et al., 2014). Gullich and Schmidtbleicher (1996) as well as Gilbert et al. (2001) reported a decrease or no change in the RFD immediately after a CA, yet a significant increase in this variable following 4 to 12 and 15 min recovery. Fatigue seems more dominant in the early stages of recovery and consequently performance of subsequent activity is diminished or unchanged. However, fatigue subsides at a faster rate than PAP, and potentiation of performance can be realized at some point during the recovery period (Tillin and Bishop, 2009). The contradictory findings related to complex training experiments suggest that the PAP-fatigue relationship and its effects on subsequent motor activity are multi-faceted. Most authors indicate that after a CA an optimal recovery time is required to diminish fatigue, realize $\mathrm{PAP}$ and maximize performance (Hodgson et al., 2005), however, there are great inconsistencies in providing the optimal recovery time (Tillin and Bishop, 2009). It has been shown that PAP is expressed sooner following a less fatiguing conditioning activity compared to a more fatiguing one (Boullosa et al., 2013). The design of this study does not allow to compare the differences between $50-130 \%$ of 1RM used in different CA. However, this study found that only the $130 \%$ and not $110 \%$ eccentric bench press showed a PAP effect, which suggests that 3 eccentric repetitions do not cause fatigue, but require apropriate exercise intensity in trained athletes.

Several authors have indicated that subjects with greater individual muscular strength show greater responses to PAP. In all 3 experiments that we conducted with competitive athletes, involving both upper and lower limbs, we observed significantly greater responses in subjects characterized by higher levels of muscular strength. This can be partially explained by fiber type distribution. The positive relationship between muscular strength and type II muscle fibers is well documented (Tillin and Bishop, 2009). It has been proposed that the RLC phosphorylation and the increase in higher order motor unit recruitment should predispose individuals with more type II muscle fibers to a greater PAP response (Baudry and Duchateu, 2007; Gilbert et al., 2001). Although subjects with a greater percentage of type II muscle fibers are expected to produce larger maximal voluntary contractions due to a higher initial anaerobic ATP turnover rate, they will also generate more metabolites associated with fatigue (Hodgson et al., 2005). This phenomenon has a direct effect on the rest interval between the CA and explosive activity. Our research also suggests that individuals with more FT fibers and consequently greater values of strength require a longer IRT.

In the group of athletic throws, we experimented with upper body PAP. Relatively few studies have examined the effect of PAP on upper body performance, yet most of these demonstrate similar effects of PAP to exercises engaging lower limbs (Ferreira et al., 2002; West et al., 2013). In our research with well-trained shot putters, javelin and discus throwers, we used the flat bench press as the CA and the bench press throw as the explosive activity. The novel aspect of this experiment included the supramaximal intensity of the CA, performed eccentrically with $110-130 \% 1 \mathrm{RM}$. We observed a nonsignificant improvement in generated power during the BP 
throw 6 min after the eccentric BP with 110\% 1RM and very significant increases in power during the BP throw when the CA was performed with $130 \%$ $1 R M$. This may suggest that extremely heavy loads used for the CA in well-trained athletes may selectively recruit FTx muscle fibers what allows for a significant effect of PAP. Previous research related to PAP demonstrates a large range of training intensities and loading parameters that have successfully been used to elicit short-term improvements in performance. However, most meta-analysis and reviews have concluded that moderate to heavier loads of $60-84 \% 1 \mathrm{RM}$ may be most effective for producing PAP (Lesinski et al., 2013; Wilson et al., 2013). Most studies conducted with competitive athletes indicate the effectiveness of higher intensities used in the CA, ranging from $70-93 \%$ 1RM (Lowery et al., 2012). Very few studies have been conducted with the use of eccentric modes of exercise with supramaximal intensity. Our results indicate significant improvement in generated power during explosive movement of the upper limbs following the flat BP performed with $130 \%$ of 1RM. Further research should be conducted with a greater range of eccentric intensities (110-180\% 1RM), evaluating changes in EMG and determining the relationship between activation, fatigue and muscle damage.

No research until now has been performed with PAP on luge athletes. Our results indicate a very significant correlation between the power generated during the Keiser Pull Down with the luge start, what justifies the use of this exercise for the CA. The PAP protocol used with luge athletes ( 3 sets of 4 reps dumbbell row at $80 \%$ $1 \mathrm{RM}$ with a $90 \mathrm{~s}$ rest interval) showed significant improvement of power during the Keiser Pull Down following $6 \mathrm{~min}$ of recovery. This suggests the possibility of PAP application in power training sessions and during competitions in luge.

The research project had several limitations. First of all the number of subjects in particular groups was rather small, what that does not allow for generalization of results. The effects of PAP could have been evaluated with a more complex methodology by using electromyography, accelerometers and kinematic analysis. In determining the optimal IRT we did not use mathematical models. The supramaximal eccentric bench press exercise modes were performed with only two intensities $(110 \%$ and $130 \%$ 1RM). Future research should concentrate on evaluating the effectiveness of eccentric contractions in the range of $110-180 \% 1 \mathrm{RM}$ and creating a mathematical model for optimizing the IRT.

\section{Conclusions and practical implications}

We confirmed the effectiveness of PAP with well-trained athletes during motor activities such as jumping, throwing and pushing/pulling. As most authors indicate, the optimal intensity of the CA for competitive athletes varies between 75$90 \%$ of 1 RM. Additionally, our research showed that eccentric supramaximal intensities $(130 \%$ $1 \mathrm{RM}$ ) can be effective in eliciting PAP in strength trained athletes. Our experiments showed that the IRT should be individualized because athletes differ in their strength level, training experience and muscle fiber structure. In the three experiments conducted with basketball players, track and field athletes and Luge athletes, the optimal IRT equaled $6 \mathrm{~min}$, yet some athletes reached peak power after $4 \mathrm{~min}$, while several others after $8 \mathrm{~min}$. This justifies the need to individualize the volume and intensity of the CA, and especially the IRT between the CA and the explosive activity.

\section{Acknowledgements}

This study was supported by research grants from Czech Science Foundation and NO. 16-13750S and by a grants of Ministry of Science and Higher Education of Poland (NRSA3 03953 and NRSA4 040 54).

\section{References}

Baechle TR, Earle RW, Wathen D. Resistance training. In: Essentials of Strength Training and Conditioning. TR 
Baechle, RW Earle, eds. Champaign, IL: Human Kinetics 2008, 381-412; 2008

Baudry S, Duchateau J. Postactivation potentiation in a human muscle: effect on the load-velocity relation of tetanic and voluntary shortening contractions. J Appl Physiol, 2007; 103(4): 1318-25

Boullosa DA, Abreu L, Beltrame LG, Behm DG. The acute effect of different half squat set configurations on jump potentiation. J Strength Cond Res, 2013; 27: 2059-2066

Chiu LZ, Fry AC, Weiss LW, Schilling BK, Brown LE, Smith SL. Postactivation potentation response in athletic and recreationally trained individuals. J Strength Cond Res, 2003; 17(4): 671-7

Czuba M, Zając A, Maszczyk A, Roczniok R, Poprzęcki S, Garbaciak W, Zając T. The Effects of High Intensity Interval Training in Normobaric Hypoxia on Aerobic Capacity in Basketball Players. J Hum Kinet, 2013; 39(1): 103-114

Docherty D, Hodgson M. The applications of postactivation potentation to elite sport. Int J Sports Physiol Perf, 2007; 2(4): 439-44

Duthie GM, Young WB, Aitken DA. The acute effects of heavy loads on jump squat performance: an evaluation of the complex and contrast methods of power development. J Strength Cond Res, 2002; 16(4): 530-8

Farup J, Sorensen H. Postactivation Potentiation: Upper Body Force Development Changes after Maximal Force Intervention. J Strength Cond Res, 2010; 24(7): 1874-1879

Ferreira SL, Panissa VL, Miarka B, Franchini E. Postactivation potentiation: effect of various recovery intervals on bench press power performance. J Strength Cond Res, 2012; 26(3): 739-44

Folland JP, Williams AG. The adaptations to strength training: morphological and neurological contributions to increased strength. Sports Med, 2007; 37(2): 145-68

French DN, Kraemer WJ, Cooke CB. Changes in dynamic exercise performance following a sequence of preconditioning isometric muscle actions. J Strength Cond Res, 2003; 17(4): 678-685

Fukunaga T, Ichionose Y, Ito M, Kawakami Y, Fukashiro S. Determination of fascicle length and pennation in a contracting human muscle in vivo. J Appl Physiol, 1997; 82(1): 354-8

Esformes JI, Bampouras TM. Effect of back squat depth on lower-body postactivation potentiation. J Strength Cond Res, 2013; 27: 2997-3000

Gilbert G, Less A, Graham-Smith P. Temporal profile of post-tetanic potentation of muscle force characteristics after repeated maximal exercise. J Sports Sci, 2001; 19: 6

Gossen ER, Sale DG. Effect of postactivation potentiation on dynamic knee extension performance. Eur J Appl Physiol, 2000; 83(6): 524-30

Gourgoulis, V, Aggeloussis, N, Kasimatis, P, Mavromatis, G, Garas A. Effect of a submaximal half-squats warm-up program on vertical jumping ability. J Strength Cond Res, 2003; 17: 342-344

Gullich A, Schmidtbleicher D. MVC-induced short-term potentation of explosive force. N Stud Athlet, 1996; 11(4): 67-81

Hamada T, Sale DG, MacDougall JD. Postactivation potentation in endurance-trained male athletes. Med. Sci. Sports Exerc, 2000; 32: 403-411

Hamada T, Sale DG. MacDougall JD, Tarnopolsky MA. Postactivation potentation, fiber type, and twitch contraction time in human knee extensor muscles. J. Appl. Physiol, 2000; 88: 2131-2137

Hodgson M, Docherty D, Robbins D. Post-activation potentation: underlying Physiology and implications for motor performance. Sports Med, 2005; 35(7): 585-95

Jensen RL, Ebben WP. Kinetic analysis of complex training rest interval effect on vertical jump performance. J Strength Cond Res, 2003, 17(2): 345-9

Lesinski M, Muehlbauer T, Büsch D, Granacher U. Acute effects of postactivation potentiation on strength and speed performance in athletes. Sportverletz Sportschaden, 2013; 27(3): 147-55

Lowery RP, Duncan NM, Loenneke JP, Sikorski EM, Naimo MA, Brown LE, Wilson FG, Wilson JM. The effects of potentiating stimuli intensity under varying rest periods on vertical jump performance and power. J Strength Cond Res, 2012; 26(12): 3320-5

(C) Editorial Committee of Journal of Human Kinetics 
Mangus BC, Takahashi M, Mercer JA, Holcomb WR, McWhorter JW, Sanchez R. Investigation of vertical jump performance after completing heavy squat exercises. J Strength Cond Res, 2006; 20(3): 597-600

Magiera A, Roczniok R, Maszczyk A, Czuba M, Kantyka J, Kurek P. The Structure of Performance of a Sport Rock Climber. J Hum Kinet, 2013; 36: 107-117

McBride JM, Nimphius S, Erickson TM. The acute effects of heavy-load squats and loaded countermovement jumps on sprint performance. J Strength Cond. Res, 2005; 19: 893-897

Sæterbakken A, van den Tillaar R, Fimland M. A comparison of muscle activity and 1-RM strength of three chest-press exercises with different stability requirements. J Sports Sci, 2011; 29(1): 1-6

Sale DG. Postactivation potentation: role in human performance. Exerc Sport Sci Rev, 2002; 30(3): 138-43

Sale DG. Postactivation potentation: role in performance. Br J Sports Med, 2004; 38(4): 386-7

van den Tillaar R, Ettema G. A comparison of muscle activity in concentric and counter movement maximum bench press. J Hum Kin, 2013; 38: 63-71

Seitz LB, de Villarreal ES, Haff GG. The temporal profile of postactivation potentiation is related to strength level. J Strength Cond Res, 2014; 28: 706-715

Seitz LB, Haff GG. Factors modulating post-activation potentiation of jump, sprint, throw, and upper-body ballistic performances: A systematic review with meta-analysis. Sports Med, 2016; 46: 231-240

Stastny P, Tufano JJ, Golas A, Petr M. Strengthening the Gluteus Medius Using Various Bodyweight and Resistance Exercises. J Strength Cond Res, 2016; 38: 91-101

Tillin NA, Bishop D. Factors modulating post-activation potentation and its effect on performance of Subsequent explosive activities. Sports Med, 2009; 39(2): 147-66

Thomas GA, Kraemer WJ, Spiering BA, Volek JS, Anderson JM, Maresh CM. Maximal Power at Different Percentages of One Repetition Maximum: Influence of Resistance and Gender. J Strength Cond Res, 2007; 21(2): 336-342

Trimble MH, Harp SS. Postexercise potentation of the H-reflex in humans. Med Sci Sports Exerc, 1998; 30(6): 933-41

Yetter M, Moir GL. The acute effects of heavy back and front squats on speed during forty-meter sprint trials. J Strength Cond Res, 2008; 22: 159-165

Young WB, Jenner A, Griffiths K. Acute enhancement of power performance from heavy load squats. $J$ Strength Cond Res, 1998; 12: 82-84

Vandervoort AA, Quinlan J, McComas AJ. Twitch potentiation after voluntary contraction. Exp. Neurol., 1983; 81: 141-152

Weber KR, Brown LE, Coburn JW, Zinder SM. Acute effects of heavy-load squats on consecutive squat jump performance. J Strength Cond Res, 2008; 22: 726-730

West DJ, Cunningham DJ, Crewther BT, Cook CJ, Kilduff LP. Influence of ballistic bench press on upper body power output in professional rugby players. J Strength Cond Res, 2013; 27(8): 2282-7

Wilson JM, Duncan NM, Marin PJ, Brown LE, Loenneke JP, Wilson SM, Jo E, Lowery RP, Ugrinowitsch C. Meta-analysis of postactivation potentation and power: effects of conditioning activity, volume, gender, rest periods and training status. J Strength Cond Res, 2013; 27(3): 854-9

Whelan N, O'Regan C, Harrison AJ. Resisted Sprints do not acutely enhance sprinting performance. $J$ Strength Cond Res, 2014; 28(7): 1858-1866

\section{Corresponding author:}

\section{Adam Zajac}

Department of Sports Training;

The Jerzy Kukuczka Academy of Physical Education in Katowice; Poland.

E-mail: a.zajac@awf.katowice.pl 